\title{
Soluble ST2 correlates with some indicators of right ventricular function in hypertensive heart failure
}

Dike B Ojji,2

Sandrine Lecour ${ }^{2}$

Olusoji M Adeyemi ${ }^{3}$

Karen Sliwa ${ }^{2,4}$

'Cardiology Unit, Department of Medicine, University of Abuja Teaching Hospital, Gwagwalada, Abuja, Nigeria; ${ }^{2}$ Department of Medicine, Faculty of Health Sciences, Hatter Institute for Cardiovascular Research in Africa, University of Cape Town, Cape Town, South Africa; ${ }^{3}$ Department of Medical Laboratory Sciences, University of Abuja Teaching Hospital, Gwagwalada, Abuja, Nigeria; ${ }^{4}$ Mary McKillop Institute for Health Research, ACU, Melbourne, VIC, Australia
Correspondence: Dike B Ojji

Cardiology Unit, Department of Medicine, University of Abuja Teaching

Hospital, Gwagwalada, Abuja, Nigeria

Tel +234806009 4456

Fax +27 21 4478789

Email dike.ojji@uniabuja.edu.ng
This article was published in the following Dove Press journal:

Vascular Health and Risk Management

16 August 2017

Number of times this article has been viewed

Purpose: ST2 receptor, which is a member of the Toll-like/interleukin-1 (IL-1) receptor family, has been found to be increased in the serum of patients 1 day after myocardial infarction. Several other studies have shown that soluble ST2 levels correlate with severity of heart failure (HF), left ventricular ejection fraction, creatinine clearance, B-type natriuretic peptide and C-reactive protein, and are predictors of mortality in HF. Most of these studies were not only limited to ischemic heart disease but also concentrated on left-sided HF. We therefore decided to study the relationship between soluble ST2 and some markers of right ventricular (RV) function in a cohort of hypertensive HF subjects.

Patients and methods: This is a prospective cohort study of hypertensive HF patients presenting to the University of Abuja Teaching Hospital, Abuja, over a 12-month period. ST2 was measured in plasma sample by the enzyme-linked immunosorbent assay (ELISA) method. Right ventricular diameters in diastole (RVDD) and right atrial area (RAA) were obtained on echocardiography, while right ventricular systolic pressure (RVSP) was estimated from echocardiography by the addition of the pressure gradient between the right ventricle and right atrium (RA) to the pressure in the RA.

Results: There was a significant correlation between RVSP and soluble ST2 $(t=0.75, p<0.0001)$, $\operatorname{RVDD}(t=0.28, p=0.004)$ and RAA $(t=0.46, p=0.002)$.

Conclusion: In a cohort of hypertensive HF subjects, soluble ST2 correlates significantly with RVSP, RVDD and RAA.

Keywords: RV function, ST2, hypertensive heart failure

\section{Introduction}

ST2 receptor is a member of the Toll-like/interleukin-1 (IL-1) receptor family with research works in animal models showing that cytokine IL-33 interacts with ST2 receptors in cardiac myocytes, thereby comprising a cardioprotective stress-responsive signaling system. ${ }^{1}$ ST2 is induced in conditions of myocardial overload, such as myocardial infarction, when the remaining viable myocardium must bear more stress. ${ }^{1}$ Soluble ST2 has been found to be increased in the serum of patients 1 day after myocardial infarction, and its serum levels predict outcome in patients with heart failure (HF). ${ }^{2}$ Several other studies have shown that soluble ST2 levels correlate with severity of HF, left ventricular ejection fraction (LVEF), creatinine clearance, B-type natriuretic peptide and C-reactive protein, and are predictors of mortality. ${ }^{3,4}$ In addition, soluble ST2 has also been found to be a useful biomarker in differentiating the various spectra of hypertensive heart disease. ${ }^{5}$ Most of these findings, however, were limited to not only ischemic heart disease but also left-sided HF. We therefore decided to study the relationship between soluble 
ST2 and some markers of right ventricular (RV) function in a cohort of hypertensive HF subjects of a population where hypertension is the commonest cause of $\mathrm{HF}^{6,7}$

\section{Patients and methods}

\section{Subjects}

This prospective cohort study was approved by the ethical clearance committee of the University of Abuja Teaching Hospital and is in compliance with the Declaration of Helsinki, and, wherever possible, it also adheres to the STROBE (STrengthening the Reporting of OBservational studies in Epidemiology) guidelines for an observational study of this kind. The minimum age for participation in the study was 18 years, but there was no upper age limit. Of the 81 patients enrolled for the study, four (4.9\%) of the total enrolment was excluded because patients were diabetic, had regional wall motion abnormality on transthoracic echocardiography, had serum creatinine levels $>170 \mu \mathrm{mol} / \mathrm{L}$ or had previous acute myocardial infarction. Therefore, 77 subjects with hypertensive HF were studied. Hypertension was defined according to the Joint National Committee VII guidelines. ${ }^{8}$ The subjects also had clinical features of long-standing hypertension, which included thickened arterial wall, locomotor brachialis and at least Grade 2 hypertensive retinopathy. HF was diagnosed according to the guidelines of the European Society of Cardiology. ${ }^{9}$ The functional status of the HF subjects was according to the guidelines of New York Heart Association Functional Classification. ${ }^{10}$ All subjects gave written informed consent to participate in the study.

\section{Questionnaire}

All the subjects completed a standard questionnaire. Owing to the multiplicity of languages in Nigeria, the questionnaire was not translated into any of the local languages. The majority of the subjects were reasonably proficient in English language. Where there was a need for interpretation, both medical and paramedical staff of the Cardiology Unit of the Department of Medicine of University of Abuja Teaching Hospital assisted. The questionnaire requested specific answers regarding date of birth, gender, occupation, background diagnosis of hypertension, background diagnosis of diabetes mellitus, history of angina pains, history of alcohol consumption and history of smoking habits.

\section{Anthropometric measurements and conventional blood pressure (BP) measurements}

The height and weight of the subjects were measured in the clinic by nursing staff with the participants standing and wear- ing indoor clothes with no shoes. Body mass index was calculated as weight in kilograms divided by the square of height in meters. Body surface area in square meter $\left(\mathrm{m}^{2}\right)$ was calculated as $(0.0001) \times(71.84) \times(\text { weight in } \mathrm{kg})^{0.425} \times(\text { height in } \mathrm{cm})^{0.725}$.

BP measurements were obtained according to the standard guidelines with a mercury sphygmomanometer (Accoson, London, UK). Systolic BP and diastolic BP were measured by cardiologists at Korotkoff sounds I and V, respectively. BP was measured at the right arm three times after a 5-minute rest with the patient in the sitting position, and the average of the three measurements was obtained. Pulse pressure was calculated as systolic BP minus diastolic $\mathrm{BP}$, while mean arterial pressure was calculated as one-third of diastolic BP plus pulse pressure.

\section{Blood measurements}

Blood samples were collected for fasting blood sugar, fasting lipid profile, electrolyte, urea and creatinine and full blood count and for NT-proBNP and ST2 assay. The blood was put into EDTA tubes, and the samples were immediately centrifuged and plasma separated and then stored at $-80^{\circ} \mathrm{C}$ until assayed. Samples were transported in dry ice and shipped to the Hatter Institute of Cardiovascular Research in Africa; the assay was carried. Details of NT-proBNP and ST2 assay have been described in our previous publication. ${ }^{11}$

\section{Transthoracic echocardiography}

Echocardiography was performed using a commercially available ultrasound system (IVIS-60). Details of echocardiography have been previously reported in a similar paper by authors. ${ }^{12}$

\section{Data analysis}

SPSS software version 16.0 (SPSS Inc., Chicago, IL, USA) was used for statistical analysis. Continuous variables were expressed as mean $\pm \mathrm{SD}$. Comparison of demographic, clinical, laboratory and echocardiographic parameters among the three groups was performed by analysis of variance. Correlation coefficients were calculated by linear regression analysis with serum NT-proBNP and soluble ST2 log transformed to establish normality, and correlations between tricuspid annular plane systolic excursion (TAPSE) and continuous demographic, clinical, laboratory and echocardiographic parameters were evaluated by Spearman regression.

\section{Results}

\section{Clinical profile of the subjects}

Table 1 shows the clinical profile of the subjects studied. The subjects were middle aged with mean age of $53.8 \pm 13.2$ years. 
Table I Clinical profile of subjects

\begin{tabular}{|c|c|c|c|c|}
\hline Parameters & All $(\mathbf{N}=77)$ & Male $(n=54)$ & Female $(n=23)$ & $p$-value \\
\hline Age, years & $53.8 \pm 13.2$ & $53.8 \pm 15.8$ & $51.7 \pm 13.6$ & 0.56 \\
\hline Smoking habits, n (\%) & $24(13.1)$ & $22(18.6)$ & $2(2.5)$ & $<0.001$ \\
\hline Body mass index, $\mathrm{kg} / \mathrm{m}^{2}$ & $24.30 \pm 7.0$ & $24.2 \pm 7.6$ & $24.5 \pm 5.9$ & 0.86 \\
\hline Palpitations, n (\%) & $40(51.9)$ & $24(44.4)$ & $16(69.6)$ & 0.002 \\
\hline Peripheral edema, n (\%) & $49(63.2)$ & $35(64.8)$ & $14(60.8)$ & NS \\
\hline \multicolumn{5}{|l|}{ NYHA class, n (\%) } \\
\hline ॥ & $14(18.2)$ & $10(18.5)$ & $4(17.5)$ & \\
\hline III & $49(63.6)$ & $35(64.8)$ & $14(60.8)$ & \\
\hline IV & $14(18.2)$ & $9(16.7)$ & $5(21.7)$ & \\
\hline $\mathrm{SBP}, \mathrm{mmHg}$ & $149.1 \pm 23.8$ & $149.9 \pm 23.8$ & $147.7 \pm 23.9$ & 0.55 \\
\hline $\mathrm{DBP}, \mathrm{mmHg}$ & $98.1 \pm 13.9$ & $98.2 \pm 13.9$ & $97.9 \pm 13.9$ & 0.92 \\
\hline $\mathrm{PP}, \mathrm{mmHg}$ & $55.8 \pm 16.2$ & $56.4 \pm 16.8$ & $54.7 \pm 15.0$ & 0.52 \\
\hline MAP, mmHg & $101.3 \pm 16.4$ & $101.2 \pm 17.2$ & $101.5 \pm 15.0$ & 0.89 \\
\hline $\mathrm{FBS}, \mathrm{mmol} / \mathrm{L}$ & $5.3 \pm 2.2$ & $5.2 \pm 2.0$ & $5.4 \pm 2.4$ & 0.58 \\
\hline $\mathrm{TC}, \mathrm{mmol} / \mathrm{L}$ & $4.2 \pm 1.2$ & $4.1 \pm 0.2$ & $4.3 \pm 1.2$ & 0.22 \\
\hline LDLC, mmol/L & $2.7 \pm 0.9$ & $2.6 \pm 1.0$ & $2.8 \pm 1.0$ & 0.14 \\
\hline $\mathrm{HDLC}, \mathrm{mmol} / \mathrm{L}$ & $1.1 \pm 0.4$ & $1.1 \pm 0.4$ & $1.1 \pm 0.3$ & 0.63 \\
\hline Estimated GFR, $\mathrm{mL} / \mathrm{min} / \mathrm{l} .73 \mathrm{~m}^{2}$ & $101.5 \pm 38.8$ & $|\mathrm{II} .6 \pm 4| .4$ & $78.3 \pm 17.0$ & $<0.0001$ \\
\hline NT-proBNP, pg/mL & $501.7 \pm 199.8$ & $513.0 \pm 208.5$ & $478.7 \pm 184.7$ & 0.58 \\
\hline Serum ST2, ng/mL & $112.9 \pm 78.7$ & $100.1 \pm 60.4$ & $134.4 \pm 98.3$ & 0.26 \\
\hline
\end{tabular}

Note: Data presented as mean \pm SD unless otherwise stated.

Abbreviations: NS, not significant; NYHA, New York Heart Association; SBP, systolic blood pressure; DBP, diastolic blood pressure; PP, pulse pressure; MAP, mean arterial pressure; FBS, fasting blood sugar; TC, total cholesterol; LDLC, low-density lipoprotein cholesterol; HDLC, high-density lipoprotein cholesterol; GFR, glomerular filtration rate.

There was no significant difference between the ages of the male and female subjects; nor was there a difference in the body mass index between the male and female subjects. Men smoked more than women $(18.6 \%$ versus $2.5 \%, p$-value $<0.0001)$ and had worse renal function with the estimated glomerular filtration rate of $111.6 \pm 41.4 \mathrm{~mL} / \mathrm{min}$ versus $78.3 \pm 17.0 \mathrm{~mL} / \mathrm{min}$ in women $(p$-value $<0.0001)$. The women presented more often with palpitations $(57.7 \%$ versus $48.9 \%$, $p$-value $=0.02$ ). Serum ST2 and NT-proBNP levels were similar in men and women.

\section{Echocardiographic profile of the subjects}

Table 2 shows the echocardiographic profile of the subjects. The male subjects had significantly more hypertrophy

Table 2 Echocardiographic profile of subjects

\begin{tabular}{|c|c|c|c|c|}
\hline Parameters & All $(\mathrm{N}=77)$ & Male $(n=54)$ & Female $(n=22)$ & $p$-value \\
\hline RVDD, cm & $3.4 \pm 0.6$ & $3.5 \pm 0.6$ & $3.2 \pm 0.5$ & 0.22 \\
\hline Left atrium, cm & $4.6 \pm 0.9$ & $4.6 \pm 0.9$ & $4.5 \pm 0.8$ & 0.17 \\
\hline IVSD, cm & $1.1 \pm 0.3$ & $1.1 \pm 0.2$ & $1.0 \pm 0.3$ & 0.03 \\
\hline PWD, cm & $1.1 \pm 0.2$ & $1.2 \pm 0.2$ & $1.1 \pm 0.2$ & 0.07 \\
\hline $\mathrm{EDD}, \mathrm{cm}$ & $5.8 \pm 1.1$ & $5.9 \pm 1.1$ & $5.5 \pm 1.1$ & 0.04 \\
\hline $\mathrm{ESD}, \mathrm{cm}$ & $4.7 \pm 1.3$ & $4.9 \pm 1.2$ & $4.5 \pm 1.3$ & 0.07 \\
\hline LAA, $\mathrm{cm}^{2}$ & $24.5 \pm 7.0$ & $24.5 \pm 6.7$ & $24.4 \pm 7.5$ & 0.95 \\
\hline $\mathrm{RAA}, \mathrm{cm}^{2}$ & $22.3 \pm 8.1$ & $22.6 \pm 8.0$ & $21.7 \pm 8.5$ & 0.50 \\
\hline LVM/HT 2.7 & $108.3 \pm 46.3$ & $117.5 \pm 35.4$ & $112.5 \pm 42.3$ & 0.65 \\
\hline LVEF, \% & $35.2 \pm 17.5$ & $34.4 \pm 16.8$ & $36.6 \pm 18.7$ & 0.58 \\
\hline $\mathrm{ME}, \mathrm{m} / \mathrm{s}$ & $0.78 \pm 0.3$ & $0.76 \pm 0.30$ & $0.8 I \pm 0.30$ & 0.43 \\
\hline $\mathrm{MA}, \mathrm{m} / \mathrm{s}$ & $0.49 \pm 0.2$ & $0.49 \pm 0.2$ & $0.49 \pm 0.1$ & 0.25 \\
\hline ME/MA & $2.2 \pm 1.3$ & $2.0 \pm 1.2$ & $2.2 \pm \mathrm{I} .4$ & 0.96 \\
\hline $\mathrm{DT}, \mathrm{ms}$ & $143.2 \pm 80.6$ & $|43.7 \pm 85|$. & $142.2 \pm 72.2$ & 0.22 \\
\hline TAPSE, mm & $16.2 \pm 5.1$ & $16.6 \pm 5.4$ & $15.5 \pm 4.5$ & 0.16 \\
\hline TAPSE $<15$ mm, n (\%) & $33(42.9)$ & $54(40.7)$ & $10(41.7)$ & 0.18 \\
\hline RVSP, mmHg & $31.4 \pm 10.5$ & $31.4 \pm 10.4$ & $31.3 \pm 10.5$ & 0.97 \\
\hline
\end{tabular}

Abbreviations: RVDD, right ventricular diameter in diastole; IVSD, interventricular septal diameter in diastole; PWD, posterior wall diameter in diastole; EDD, end-diastolic diameter; ESD, end-systolic diameter; LAA, left atrial area; RAA, right atrial area; LVM, left ventricular mass; HT, height; LVEF, left ventricular ejection fraction; ME, early mitral inflow; MA, late mitral inflow; DT, deceleration time; TAPSE, tricuspid annular plane systolic excursion; RVSP, right ventricular systolic pressure. 
involving the interventricular septal wall compared to the female subjects. They also had a more dilated left ventricle (LV), especially in diastole, compared to the female subjects. There was no gender difference in the LV mass indexed for height. ${ }^{2,7}$ Using TAPSE, 33 (42.9\%) of the subjects had impaired RV systolic function.

\section{Relationship between TAPSE and echocardiographic and laboratory parameters}

Table 3 shows the relationship between RV systolic function as assessed by TAPSE and various echocardiographic and laboratory parameters. Left atrial diameter, areas of both atria, LV end-diastolic and end-systolic diameters, LV mass index, right ventricular systolic pressure (RVSP), LVEF and transmitral $E / A$ ratio correlated significantly with TAPSE. There was no significant correlation between TAPSE and cardiac biomarkers.

\section{Univariate association between log- transformed ST2 and echocardiographic right-sided parameters}

Table 4 shows that soluble ST2 correlates significantly with RVSP, right ventricular diameter in diastole (RVDD) and right atrial area (RAA), while NT-proBNP only correlates significantly with RVSP and RAA.

\section{Discussion}

Our study has shown a correlation between soluble ST2 and RVSP, RVDD and RAA, supporting a previous report in a cohort of HF secondary to ischemic heart disease and idiopathic dilated cardiomyopathy. ${ }^{13}$ Therefore, the relationship between ST2 and RVSP is not only restricted to both ischemic heart disease and idiopathic dilated cardiomyopathy but extends to hypertensive HF. We did not however find any correlation between soluble ST2 and tricuspid annular plane excursion. This finding has to be further verified in
Table 3 Univariate analysis with RV systolic function using TAPSE

\begin{tabular}{lll}
\hline Parameters & Pearson correlation & p-value \\
\hline Left atrial diameter & -0.54 & $<0.0001$ \\
EDD & -0.47 & $<0.000$ I \\
ESD & -0.55 & $<0.000$ I \\
LVM/HT2.7 & -0.34 & $<0.000$ I \\
LVEF & 0.60 & $<0.000$ I \\
ME/MA & -0.39 & $<0.0001$ \\
Left atrial area & -0.24 & 0.02 \\
RAA & -0.44 & $<0.0001$ \\
RVSP & -0.74 & $<0.0001$ \\
RVDD & -0.035 & 0.77 \\
Soluble ST2 & 0.04 & 0.68 \\
NT-proBNP & 0.000 & 0.99 \\
\hline
\end{tabular}

Abbreviations: RV, right ventricular; TAPSE, tricuspid annular plane systolic excursion; EDD, end-diastolic diameter; ESD, end-systolic diameter; HT, height; LVM, left ventricular mass; LVEF, left ventricular ejection fraction; ME, early mitral inflow; MA, late mitral inflow; RAA, right atrial area; RVSP, right ventricular systolic pressure; RVDD, right ventricular diameter in diastole.

a larger cohort. Although NT-proBNP correlated well with RVSP and RAA, there was poor correlation with RVDD, which may suggest that soluble ST2 is a better circulating biomarker than the more traditional NT-proBNP in assessing hypertensive HF.

On linear regression analysis, factors that were associated with abnormal RV function included dilated left atrium and LV, increased LV mass, reduced LV systolic function, restrictive filling pattern and RVSP. LV dilation has been previously shown to have an impact on RV function as measured by TAPSE.${ }^{14}$ It has been previously shown that a reduced LVEF impacts on TAPSE even in the setting of preserved RV ejection fraction. ${ }^{14,15}$ The effect of LVEF on TAPSE has been attributed to ventricular interdependence. ${ }^{16}$

The finding in our study of an inverse relationship between RV systolic function and RVSP is in accordance with most previous studies. ${ }^{17}$ This relationship has been reported in patients with dilated cardiomyopathy and ischemic heart diseases, supporting the fact that the major determinant of RV systolic function in left-sided HF is after load mismatch due to increase in pulmonary artery resistance. ${ }^{17}$ TAPSE

Table 4 Univariate and multivariate associations between log-transformed ST2 and echocardiography right-sided parameters

\begin{tabular}{|c|c|c|c|c|}
\hline \multicolumn{3}{|c|}{ Univariate association } & \multicolumn{2}{|l|}{ Multivariate association } \\
\hline Parameters & Serum ST2, RI & NT-proBNP, R2 & Standard coefficient $(\beta)$ & $p$-value \\
\hline$\overline{R V S P}$ & $0.75^{*}$ & $0.54 * *$ & 0.46 & $<0.001$ \\
\hline RAA & $0.46 *$ & $0.20 * *$ & 034 & 0.003 \\
\hline RVDD & $0.28 *$ & 0.09 & - & - \\
\hline TAPSE & -0.15 & -0.13 & - & - \\
\hline
\end{tabular}

Notes: RI, coefficient of correlation between soluble ST2 and variables; R2, coefficient of correlation between NT-proBNP and variables. ${ }^{*} p<0.05$ versus soluble $S T 2$. $* * p<0.05$ versus NT-proBNP.

Abbreviations: RVSP, right ventricular systolic pressure; RAA, right atrial area; RVDD, right ventricular diameter in diastole; TAPSE, tricuspid annular plane systolic excursion. 
correlated with markers of diastolic dysfunction and RVSP in our study but not with RV end-diastolic diameter as previously reported. ${ }^{18}$

We found RV systolic dysfunction (measured by TAPSE) in 33 of the 77 subjects studied. The high prevalence of RV systolic dysfunction in our hypertensive HF cohort might partly explain the poor prognosis that is found in our subjects, ${ }^{19}$ as RV systolic dysfunction is a poor prognostic factor in HF. ${ }^{16,17}$ There is a need therefore to pay more attention to RV function, which has often been neglected in hypertensive HF subjects. The impairment of RV function in left-sided HF has been linked to ventricular interdependence, ${ }^{18}$ which is always present in HF, being most apparent with changes in loading conditions such as those seen with volume loading. ${ }^{15}$

\section{Limitation}

Since our study is limited by the number of subjects, the relationship between plasma ST2 and echocardiographic variables seen in this study should be viewed as hypothesis generating for studies in larger patient cohorts. In addition, RV function was only assessed by TAPSE and not by other parameters such as TAPSE/pulmonary arterial systolic pressure (PAPS) and fractional area change (FAC). In addition, since the diagnosis of ischemic heart was made clinically using history, troponin I levels and electrocardiography with no myocardial perfusion imaging and coronary angiography performed, it is possible that subtle coronary artery disease might have been missed, which is a confounding factor as ischemic heart disease increases the concentration of soluble ST2. ${ }^{2,3}$ However, the careful and extensive phenotyping of our study population with respect to clinical, echocardiographic and biochemical parameters must have reduced the effect of these limitations and is, therefore, a strong point in this study.

\section{Conclusion}

A higher concentration of soluble ST2 correlates with higher RVSP in a cohort of hypertensive HF. Therefore, the association of ST2 with RVSP is not only limited to HF due to ischemic heart disease and idiopathic dilated cardiomyopathy but also HF secondary to hypertensive heart disease.

\section{Acknowledgments}

Our sincere appreciation goes to all members of staff of Hatter Institute for Cardiovascular Research in Africa, Department of Medicine, Faculty of Health Sciences, University of Cape Town, South Africa, and members of staff of Cardiology Unit, Department of Medicine, University of Abuja Teaching
Hospital, Gwagwalada, Abuja. The project was supported by Award Number D43TW008330 from the Fogarty International Center. The content is solely the responsibility of the authors and does not necessarily represent the official view of the Fogarty International Center or the National Institutes of Health. The authors would like to acknowledge the funding source for the biomarker assays: National Research Foundation South Africa, SERVIER and the Maurice Hatter Foundation. The abstract of this paper was presented at the World Congress of Cardiology, June 2016, in Mexico City, Mexico, as a poster presentation. We found that in a cohort of hypertensive HF subjects, soluble ST2 correlated significantly with RVSP, RVDD and right atrial. The poster's abstract was published in Journal of Global Health 2016.

\section{Author contributions}

DBO designed the review, conducted the literature search and drafted the first version of the manuscript. All authors contributed toward data analysis, drafting and critically revising the paper and agree to be accountable for all aspects of the work.

\section{Disclosure}

The authors report no conflicts of interest in this work.

\section{References}

1. Sanada S, Hakuno D, Higgins LJ, Schreiter ER, McKenzie AN, Lee RT. IL-33 and ST2 comprise a critical biomechanically induced and cardio protective signaling system. J Clin Invest. 2007;117(6):1538-1549.

2. Weinberg EO, Shimpo M, De Keulenaer GW, et al. Expression and regulation of ST2, an interleukin-1 receptor family member, in cardiomyocytes and myocardial infarction. Circulation. 2002;106(23):2961-2967.

3. Mueller T, Dieplinger B, Gegenhuber A, Poelz W, Pacher R, Haltmayer M. Increased plasma concentrations of soluble ST2 are predictive for 1-year mortality in patients with acute destabilized heart failure. Clin Chem. 2008;54(4):752-756.

4. Daniels LB, Clopton P, Iqbal N, Tran K, Maisel AS. Association of ST2 levels with cardiac structure and function and mortality in outpatients. Am Heart J. 2010;160(4):721-728.

5. Ojji DB, Opie LH, Lecour S, Lacerda L, Adeyemi OM, Sliwa K. The effect of left ventricular remodelling on soluble ST2 in a cohort of hypertensive subjects. J Hum Hypertens. 2014;28(7):432-437.

6. Damasceno A, Mayosi BM, Ojji DB, et al. The causes, treatment and outcome of acute heart failure in 1006 Africans from 9 countries. Arch Intern Med. 2012;172(18):1386-1394.

7. Ojji D, Stewart S, Ajayi S, Manmak M, Sliwa K. A predominance of hypertensive heart failure in the Abuja Heart Study Cohort of Urban Nigerians: a prospective clinical registry of 1515 de novo cases. Eur $J$ Heart Fail. 2013;15(8):835-842.

8. US Department of Health and Human Sciences. The seventh report of the Joint National Committee on prevention, evaluation and treatment of high blood pressure. NIH Publ. 2004;04:5230.

9. Swedberg K, Cleland J, Dargie H, et al; Task Force for the Diagnosis and Treatment of Chronic Heart Failure of the European Society of Cardiology. The task force for the diagnosis and treatment of chronic heart failure of the European Society of Cardiology: guidelines for the treatment and diagnosis of chronic heart failure: an executive summary (update 2005). Eur Heart J. 2005;26(11):1115-1140. 
10. Criteria Committee, New York Heart Association. Diseases of Heart and Blood Vessels. Nomenclature and Criteria for Diagnosis. 6th ed. Boston, MA: Little, Brown \& Co; 1964:114.

11. Ojji DB, Opie LH, Lecour S, Lacerda L, Adeyemi O, Sliwa K. Relationship between left ventricular geometry and soluble ST2 in a cohort of hypertensive patients. J Clin Hypertens. 2013;15(12):899-904.

12. Ojji DB, Lecour S, Atherton JJ, Blauwet LA, Alfa J, Sliwa K. Right ventricular systolic dysfunction is common in hypertensive heart failure: a prospective study in Sub-Saharan Africa. PLoS One. 2016;11(4):e0153479.

13. Drazner MH, Dries DL, Peshock RM, et al. Serum levels of the interleukin-1 receptor family member ST2, cardiac structure and function, and long term mortality in patients with acute dyspnoea. Circ Heart Fail. 2009;2(4):311-319.

14. JuilliereY, Buffer P, Marie PY, BerderV, Danchin N, Cherrier F. Comparison of right ventricular systolic function in idiopathic dilated cardiomyopathy and healed anterior wall myocardial infarction associated with atherosclerotic coronary artery disease. Am J Cardiol. 1994;73(8):588-590.
15. Atherton JJ, Moore TD, Lele SS, et al. Diastolic ventricular interaction in chronic heart failure. Lancet. 1997;349(9067):1720-1724.

16. Jurcut R, Giusca S, La Gerche A, Vasile S, Ginghina C, Voigt JU. The echocardiographic assessment of the right ventricle: what to do in 2010 ? Eur J Echocardiogr. 2010;11(2):81-96.

17. Isezuo AS, Omotoso AB, Araoye MA, Carr J, Corrah T. Determinants of prognosis among black Africans with hypertensive heart failure. Afr J Med Med Sci. 2003;32(2):143-149.

18. Lopez-Candales A, Rajagopalan N, Saxena N, et al. Right ventricular systolic function is not the sole determinant of tricuspid annular motion. Am J Cardiol. 2006;98(7):973-977.

19. De Groote P, Millaire A, Foucher-Hossein C, et al. Right ventricular ejection fraction is an independent predictor of survival in patients with moderate heart failure. J Am Coll Cardiol. 1998;32(4): 948-954.

\section{Publish your work in this journal}

Vascular Health and Risk Management is an international, peerreviewed journal of therapeutics and risk management, focusing on concise rapid reporting of clinical studies on the processes involved in the maintenance of vascular health; the monitoring, prevention and treatment of vascular disease and its sequelae; and the involvement of metabolic disorders, particularly diabetes. This journal is indexed on PubMed Central and MedLine. The manuscript management system is completely online and includes a very quick and fair peer-review system, which is all easy to use. Visit http://www.dovepress.com/ testimonials.php to read real quotes from published authors. 